Pure and Applied Mathematics Quarterly

Volume 5, Number 2

(Special Issue: In honor of

Friedrich Hirzebruch, Part 1 of 2)

$729-753,2009$

\title{
Eta Invariants and Class Numbers
}

\author{
Roberto J. Miatello and Ricardo A. Podestá
}

\begin{abstract}
Let $M$ be a compact flat spin Riemannian manifold, having cyclic holonomy group of odd prime order $p$. If $D$ is the Dirac operator acting on spinor fields of $M$, we give explicit expressions for the eta series $\eta(s)$ and the eta invariant $\eta=\eta(0)$. We prove that $\eta(s)=e(s) L(s, \chi)$ where $e(s)$ is a linear combination of exponentials and $L(s, \chi)$ is the Dirichlet $L$-function attached to $\chi(k)=\left(\frac{k}{p}\right)$, the Legendre symbol.

Furthermore, for $p \neq 3$, we show that $\eta$ is an explicit integral multiple of the class number $h_{-p}$ of the imaginary quadratic field $\mathbb{Q}(\sqrt{-p})$. We also provide alternative expressions for $\eta$ as finite cotangent or cosecant sums.
\end{abstract}

Keywords: Eta invariants and eta series, compact flat manifolds, spin structures, Dirac operator, class numbers

\section{INTRODUCTION}

If $A$ is a self-adjoint elliptic differential operator on a compact $n$-manifold $M$, then $A$ has a discrete spectrum, denoted by $\operatorname{Spec}_{A}(M)$, consisting of real eigenvalues $\lambda$ with finite multiplicity $d_{\lambda}$. The spectrum is said to be asymmetric if for some $\lambda \in \operatorname{Spec}_{A}(M)$ one has that $d_{\lambda} \neq d_{-\lambda}$. To study this phenomenon, Atiyah, Patodi and Singer introduced ([2]) the eta series

$$
\eta_{A}(s)=\sum_{0 \neq \lambda \in \text { Spec }_{A}} \operatorname{sign}(\lambda)|\lambda|^{-s} .
$$

Received January 3, 2006.

2000 Mathematics Subject Classification. Primary 58J53; Secondary 58C22, 20H15.

Supported by Conicet and SECyT-UNC 
This series converges for $\operatorname{Re}(s)>\frac{n}{d}$, where $d$ is the order of $A$, and defines a holomorphic function $\eta_{A}(s)$ which has a meromorphic continuation to $\mathbb{C}$ having (possibly) simple poles at $s=n-k$, with $k \in \mathbb{N}_{0}$. It is a remarkable fact that $\eta_{A}(s)$ is finite at $s=0$, i.e. its residue vanishes at the origin (see [2] for $n$ odd, [11] for $n$ even). The number $\eta_{A}(0)$ is a non-local spectral invariant, called the $\eta$-invariant. It gives a measure of the spectral asymmetry of $A$.

In this paper we take $A$ to be the Dirac operator $D$, which is a first order elliptic essentially self-adjoint operator defined on sections of the spinor bundle over a spin manifold $M$. It is known that if $n \not \equiv 3(\bmod 4)$ then $\eta(s) \equiv 0$ (see $[\mathbf{9}]$ ), thus we shall only consider manifolds of odd dimension $n=4 r+3$. The determination of the associated eta function $\eta(s)$ and of the eta invariant $\eta(0)$ is in general a difficult task and the explicit computation has been carried out for a very small class of Riemannian manifolds. The aim of this paper is to give explicit expressions of these spectral invariants for compact flat manifolds with cyclic holonomy group $\mathbb{Z}_{p}, p$ an odd prime, and to show connections with classical number theory. Any compact flat manifold is isometric to a quotient $M_{\Gamma}:=\Gamma \backslash \mathbb{R}^{n}$, with $\Gamma$ a Bieberbach group. If $\Lambda$ denotes the translation lattice of $\Gamma$, then $F=\Lambda \backslash \Gamma$ is a finite group, the Riemannian holonomy group of $M_{\Gamma}$. In the terminology adopted by Charlap $([\mathbf{6}])$, a compact flat manifold having cyclic holonomy group isomorphic to $\mathbb{Z}_{p}$ will be called a $\mathbb{Z}_{p}$-manifold. Any Riemannian manifold with holonomy group $\mathbb{Z}_{p}$ is necessarily flat, hence of the form $M_{\Gamma}$ as above.

We will explicitly compute the spectral invariants $\eta(s)$ and $\eta$ for an arbitrary $\mathbb{Z}_{p}$-manifold, for every odd prime $p$ and any dimension $n$. Our main tools are the formulas in [17] giving the multiplicity of the eigenvalues of the Dirac operator $D$ (see (2.9) in Theorem 2.5), together with expressions for variations of classical character Gauss sums (see the Appendix). We shall also make use of the known classification of $\mathbb{Z}_{p}$-manifolds (due to Charlap ([6] )) that will make it possible to get a general result for eta invariants in the present case. The manifolds involved will correspond exactly to those Bieberbach groups called exceptional in [7].

In one of the main results of this paper, we will compute the eta series $\eta(s)$ for an arbitrary $\mathbb{Z}_{p}$-manifold. For those $\mathbb{Z}_{p}$-manifolds that may possibly have asymmetric Dirac spectrum, and for the two existing spin structures $\varepsilon_{1}, \varepsilon_{2}$, we will prove that $\eta(s)=e(s) L(s, \chi)$, with $e(s)$ a linear combination of exponentials, 
$L(s, \chi)$ a Dirichlet $L$-function and $\chi$ the quadratic character associated to the Legendre symbol (see Theorem 3.3). Alternatively, they can be written as linear combinations of differences of Hurwitz zeta functions, with coefficients given by Legendre symbols (see Corollary 3.4). In particular, this implies that $\eta(s)$ is entire.

In Section 4, using the expressions of $\eta(s)$, we compute the corresponding $\eta$-invariants, expressing them in terms of class numbers of imaginary quadratic fields. Indeed, let $\mathbb{Q}\left(\xi_{p}\right)$ be the cyclotomic field with $\xi_{p}$ a primitive $p^{\text {th }}$-root of unity, with $p$ an odd prime. One has that $\mathbb{Q}\left(\xi_{p}\right)$ contains the quadratic number field $\mathbb{Q}(i \sqrt{p})=\mathbb{Q}(\sqrt{-p})$. Let $h_{-p}$ and $\omega_{-p}$ denote respectively the class number and the number of roots of unity in $\mathbb{Q}(\sqrt{-p})$. In Theorem 4.1 we show that, if $n=a(p-1)+1$ with $a$ odd and $p=4 t+3$, then

$$
\eta_{\varepsilon_{1}}=(-1)^{r+t+1} 4 p^{\frac{a-1}{2}} \frac{h_{-p}}{\omega_{-p}} \quad \text { and } \quad \eta_{\varepsilon_{2}}= \begin{cases}0 & p \equiv 7(\bmod 8) \\ -2 \eta_{\varepsilon_{1}} & p \equiv 3(\bmod 8) .\end{cases}
$$

where $r=\frac{n-3}{4}$. We note that $\omega_{-p}=6$ for $p=3$ and $\omega_{-p}=2$ for any $p>3$.

In the case $n=p=3$, this gives $\eta_{\varepsilon_{1}}=-\frac{2}{3}$ and $\eta_{\varepsilon_{2}}=\frac{4}{3}$, in coincidence with the values obtained by Pfäffle $([\mathbf{1 8}])$.

We also give alternative expressions for $\eta_{\varepsilon_{1}}, \eta_{\varepsilon_{2}}$, as finite cotangent or cosecant sums with coefficients involving Legendre symbols (see Proposition 4.3).

At the end of Section 4, we show how to get the formulas for the $\eta$-invariants involving class numbers, directly from the expressions involving trigonometric functions (4.4) and (4.5). This argument was pointed out to the first author by Prof. F. Hirzebruch during a stay at the M.P.I.M. The authors wish to thank Prof. Hirzebruch for his help.

\section{Preliminaries}

Compact flat manifolds. We first review some standard facts on compact flat manifolds (see [6] or $[\mathbf{2 3}]$ ). A Bieberbach group is a discrete, cocompact, torsionfree subgroup $\Gamma$ of $\mathrm{I}\left(\mathbb{R}^{n}\right)$, the isometry group of $\mathbb{R}^{n}$. Such $\Gamma$ acts properly discontinuously on $\mathbb{R}^{n}$, thus $M_{\Gamma}=\Gamma \backslash \mathbb{R}^{n}$ is a compact flat Riemannian manifold with fundamental group $\Gamma$. Any such manifold arises in this way. Any element $\gamma \in \mathrm{I}\left(\mathbb{R}^{n}\right)=\mathrm{O}(n) \rtimes \mathbb{R}^{n}$ decomposes uniquely as $\gamma=B L_{b}$, where $B \in \mathrm{O}(n)$ and 
$L_{b}$ denotes translation by $b \in \mathbb{R}^{n}$. The translations in $\Gamma$ form a normal, maximal abelian subgroup of finite index, $L_{\Lambda}, \Lambda$ a lattice in $\mathbb{R}^{n}$ that is $B$-stable for each $B L_{b} \in \Gamma$. The restriction to $\Gamma$ of the canonical projection $r: \mathrm{I}\left(\mathbb{R}^{n}\right) \rightarrow \mathrm{O}(n)$ given by $B L_{b} \mapsto B$ is a homomorphism with kernel $L_{\Lambda}$ and $F:=r(\Gamma)$ is a finite subgroup of $\mathrm{O}(n)$. The group $F \simeq \Lambda \backslash \Gamma$ is called the holonomy group of $\Gamma$ and gives the linear holonomy group of the Riemannian manifold $M_{\Gamma}$. We shall assume throughout this paper that $M_{\Gamma}$ is orientable, i.e. $F \subset \mathrm{SO}(n)$. The action by conjugation of $\Lambda \backslash \Gamma$ on $\Lambda$ defines an integral representation of $F$, called the integral holonomy representation.

For $\Lambda$ a lattice and $\mu \geq 0$, we put $\Lambda_{\mu}=\{\lambda \in \Lambda:\|\lambda\|=\mu\}$. Also, if $B \in \mathrm{O}(n)$ we set $\Lambda^{B}=\{\lambda \in \Lambda: B \lambda=\lambda\},\left(\Lambda_{\mu}\right)^{B}=\Lambda_{\mu} \cap \Lambda^{B}$, and

$$
n_{B}:=\operatorname{dim} \operatorname{ker}(B-I d)=\operatorname{dim}\left(\mathbb{R}^{n}\right)^{B} .
$$

If $\Gamma$ is a Bieberbach group then the torsion-free condition implies that $n_{B}>0$ for any $\gamma=B L_{b} \in \Gamma$.

Spin group. Let $C l(n)$ denote the Clifford algebra of $\mathbb{R}^{n}$ with respect to the standard inner product. If $e_{1}, \ldots, e_{n}$ is the canonical basis of $\mathbb{R}^{n}$ then $C l(n)$ has basis $\left\{e_{i_{1}} \cdots e_{i_{j}}: i_{1}<\cdots<i_{j}\right\}$, with $e_{i} e_{j}=-e_{j} e_{i}$ for $i \neq j$ and $e_{i}^{2}=-1$ for $1 \leq i \leq n$. Inside the group of units of $C l(n)$ we have the spin group given by $\operatorname{Spin}(n)=\left\{g=v_{1} \cdots v_{2 k}:\left\|v_{j}\right\|=1, j=1, \ldots, 2 k\right\}$ which is a compact, simply connected Lie group if $n \geq 3$. There is a canonical epimorphism

$$
\mu: \operatorname{Spin}(n) \rightarrow \mathrm{SO}(n)
$$

given by $v \mapsto\left(x \mapsto v x v^{-1}\right)$ with kernel $\{ \pm 1\}$. It is easy to check that, for $t_{1}, \ldots, t_{m} \in \mathbb{R}, m=\left\lfloor\frac{n}{2}\right\rfloor$, the elements

$$
x\left(t_{1}, \ldots, t_{m}\right):=\prod_{j=1}^{m}\left(\cos t_{j}+\sin t_{j} e_{2 j-1} e_{2 j}\right) \in \operatorname{Spin}(n)
$$

satisfy $x\left(t_{1}+\pi, t_{2}, \ldots, t_{m}\right)=-x\left(t_{1}, t_{2}, \ldots, t_{m}\right)$ and, for $k \in \mathbb{Z}$, also

$$
x\left(t_{1}, \ldots, t_{m}\right)^{k}=x\left(k t_{1}, \ldots, k t_{m}\right) .
$$

For convenience, if $a \in \mathbb{N}$, we shall use the notation

$$
x_{a}\left(t_{1}, t_{2}, \ldots, t_{s}\right):=x(\underbrace{t_{1}, t_{2}, \ldots, t_{s}}_{1}, \underbrace{t_{1}, t_{2}, \ldots, t_{s}}_{2}, \ldots, \underbrace{t_{1}, t_{2}, \ldots, t_{s}}_{a}) .
$$


Now, let $B(t)=\left[\begin{array}{c}\cos t-\sin t \\ \sin t \\ \cos t\end{array}\right]$ with $t \in \mathbb{R}$. For $t_{1}, \ldots, t_{m} \in \mathbb{R}$, if $n=2 m+1$, set $x_{0}\left(t_{1}, \ldots, t_{m}\right):=\operatorname{diag}\left(B\left(t_{1}\right), \ldots, B\left(t_{m}\right), 1\right)$, and omit the 1 if $n=2 m$. Maximal tori in $\operatorname{Spin}(n)$ and $\mathrm{SO}(n)$ are respectively given by $T=\left\{x\left(t_{1}, \ldots, t_{m}\right): t_{j} \in \mathbb{R}\right\}$ and $T_{0}=\left\{x_{0}\left(t_{1}, \ldots, t_{m}\right): t_{j} \in \mathbb{R}\right\}$. The restriction $\mu: T \rightarrow T_{0}$ is a 2 -fold cover and we have

$$
\mu\left(x\left(t_{1}, \ldots, t_{m}\right)\right)=x_{0}\left(2 t_{1}, \ldots, 2 t_{m}\right)
$$

Spin representations. Consider $\left(L_{n}, \mathrm{~S}_{n}\right)$ an irreducible complex representation of the complexified algebra $C l(n) \otimes \mathbb{C}$, restricted to $\operatorname{Spin}(n)$. The complex vector space $\mathrm{S}_{n}$ has dimension $2^{m}$ with $m=\left[\frac{n}{2}\right]$. If $n$ is odd, then $\left(L_{n}, \mathrm{~S}_{n}\right)$ is irreducible for $\operatorname{Spin}(n)$ and is called the spin representation. If $n$ is even, then $\mathrm{S}_{n}=\mathrm{S}_{n}^{+} \oplus \mathrm{S}_{n}^{-}$ where each $\mathrm{S}_{n}^{ \pm}$is irreducible of dimension $2^{m-1}$. The representations $L_{n}^{ \pm}:=L_{n \mid S_{n}^{ \pm}}$ are called the half-spin representations. If $n=2 m$, the values of the characters $\chi_{L^{ \pm}}$of the half-spin representations on the torus $T$ are given by (see [17], Lemma $6.1)$

$$
\chi_{L_{n}^{ \pm}}\left(x\left(t_{1}, \ldots, t_{m}\right)\right)=2^{m-1}\left(\prod_{j=1}^{m} \cos t_{j} \pm i^{m} \prod_{j=1}^{m} \sin t_{j}\right)
$$

Spin structures on flat manifolds. It is a well known fact (see [9], [14] or [18]) that if $M$ is a compact flat spin manifold, the spin structures on $M$ are in a one-to-one correspondence with group homomorphisms

$$
\varepsilon: \Gamma \rightarrow \operatorname{Spin}(n) \quad \text { such that } \quad \mu \circ \varepsilon=r,
$$

where $r(\gamma)=B$ if $\gamma=B L_{b} \in \Gamma$ and $\mu$ is as in (1.2). Throughout the paper we shall denote by $\left(M_{\Gamma}, \varepsilon\right)$ a spin Bieberbach manifold endowed with the spin structure induced by $\varepsilon$.

We recall that the $n$-torus admits $2^{n}$ spin structures $([\mathbf{8}])$, while a general flat manifold $M_{\Gamma}$ need not admit any $([\mathbf{3}],[\mathbf{1 5}],[\mathbf{1 3}],[\mathbf{1 6}])$.

Spectrum of the Dirac operator. Consider the vector bundle $S\left(M_{\Gamma}, \varepsilon\right):=\Gamma \backslash\left(\mathbb{R}^{n} \times\right.$ S) $\rightarrow \Gamma \backslash \mathbb{R}^{n}$ where the action of $\Gamma$ is given by

$$
\gamma \cdot(x, w)=\left(\gamma x, L_{n}(\varepsilon(\gamma))(w)\right), \quad \text { for } \gamma \in \Gamma, w \in \mathrm{S}_{n} .
$$

Then $S\left(M_{\Gamma}, \varepsilon\right)$ is called the spinor bundle of $M_{\Gamma}$. The space $\Gamma^{\infty}\left(S\left(M_{\Gamma}, \varepsilon\right)\right)$ of smooth sections of $S\left(M_{\Gamma}, \varepsilon\right)$ can be identified with the space of smooth functions $f: \mathbb{R}^{n} \rightarrow$ S such that $f(\gamma x)=L_{n}(\varepsilon(\gamma))(f(x))$. 
The Dirac operator $D$ acts on smooth sections $f$ of the spinor bundle $S\left(M_{\Gamma}, \varepsilon\right)$ by $D f(x)=\sum_{i=1}^{n} e_{i} \frac{\partial f}{\partial x_{i}}(x)$ where $e_{i}$ acts by $L_{n}\left(e_{i}\right)$ on $\mathrm{S}_{n}$. One has that $D$ is an elliptic first-order differential operator, symmetric and essentially self-adjoint. Furthermore, since $M$ is compact, $D$ has a discrete spectrum consisting of real eigenvalues $\pm 2 \pi \mu$, of finite multiplicity $d_{\mu}^{ \pm}$. In [17], Theorem 2.5, we obtained explicit expressions for the multiplicities $d_{\mu}^{ \pm}$for any compact flat spin manifold $\left(M_{\Gamma}, \varepsilon\right)$ with translation lattice $\Lambda$ and holonomy group $F$.

For $n$ odd and $\mu>0$, we showed that the multiplicities are given by

$$
\begin{aligned}
d_{\mu}^{ \pm}(\Gamma, \varepsilon)=\frac{1}{|F|}\left(\sum_{\substack{\gamma=B L_{b} \in \Lambda \backslash \Gamma \\
B \notin F_{1}}} \sum_{u \in\left(\Lambda_{\varepsilon, \mu}^{*}\right)^{B}} e^{-2 \pi i u \cdot b} \chi_{L_{n-1}^{ \pm}}\left(x_{\gamma}\right)+\right. \\
\left.\sum_{\substack{\gamma=B L_{b} \in \Lambda \backslash \Gamma \\
B \in F_{1}}} \sum_{u \in\left(\Lambda_{\varepsilon, \mu}^{*}\right)^{B}} e^{-2 \pi i u \cdot b} \chi_{\substack{L_{n-1}^{ \pm \sigma\left(u, x_{\gamma}\right)}\\
}}\left(x_{\gamma}\right)\right) .
\end{aligned}
$$

The terms in the formula are defined as follows. First, $F_{1}=\left\{B \in F: n_{B}=1\right\}$ and, for each $\gamma=B L_{b} \in \Gamma,\left(\Lambda_{\varepsilon, \mu}^{*}\right)^{B}$ denotes the set of elements of norm $\mu$ fixed by $B$ in $\Lambda_{\varepsilon}^{*}=\left\{u \in \Lambda^{*}: \varepsilon\left(L_{\lambda}\right)=e^{2 \pi i \lambda \cdot u}, \lambda \in \Lambda\right\}$. If $\lambda_{1}, \ldots, \lambda_{n}$ is a $\mathbb{Z}$-basis of $\Lambda$ denote by $\lambda_{1}^{\prime}, \ldots, \lambda_{n}^{\prime}$ the dual basis and put $J_{\varepsilon}^{ \pm}:=\left\{i \in\{1, \ldots, n\}: \varepsilon\left(L_{\lambda_{i}}\right)= \pm 1\right\}$. We then have

$$
\Lambda_{\varepsilon}^{*}=\bigoplus_{j \in J_{\varepsilon}^{+}} \mathbb{Z} \lambda_{j}^{\prime} \oplus \bigoplus_{j \in J_{\varepsilon}^{-}}\left(\mathbb{Z}+\frac{1}{2}\right) \lambda_{j}^{\prime} .
$$

Furthermore, for $\gamma \in \Gamma, x_{\gamma}$ is a fixed element in the maximal torus of $\operatorname{Spin}(n-$ $1)$, conjugate in $\operatorname{Spin}(n)$ to $\varepsilon(\gamma)$. Finally, $\sigma\left(u, x_{\gamma}\right)$ is a sign, depending on $u$ and on the conjugacy class of $x_{\gamma}$ in $\operatorname{Spin}(n-1)$ (see Definition 2.3, Remark 2.4 and Lemma 6.2 in $[\mathbf{1 7}]$ for details).

\section{2. $\mathbb{Z}_{p}$-MANIFOLDS}

Our main goal in this paper will be to obtain several explicit expressions for the eta series and eta invariants of an arbitrary $\mathbb{Z}_{p}$-manifold, $p$ an odd prime. Using the classification given by Charlap in [6], we shall first describe the $\mathbb{Z}_{p}$-manifolds that may possibly have asymmetric Dirac spectrum. For such manifolds, we shall compute the eta series $\eta(s)$ explicitly, proving that, up to a multiple, it is given by 
a Dirichlet $L$-function $L(s, \chi)$, where $\chi$ is the Legendre symbol. As a consequence, we show that $\eta(s)$ can also be written as a linear combination of differences of Hurwitz zeta functions, with Legendre symbols as coefficients. Such expressions automatically imply that $\eta(s)$ is everywhere holomorphic in $\mathbb{C}$.

We shall first recall Charlap's classification of Bieberbach groups with holonomy group $\mathbb{Z}_{p}, p$ an odd prime (see $[\mathbf{6}]$ or $[\mathbf{7}]$ ). The $\mathbb{Z}_{p}$-modules of rank $n$ were classified by Reiner in $[\mathbf{2 0}]$, who showed that the integral representations of $\mathbb{Z}_{p}$ have the form

$$
\Lambda=\mathfrak{a} \oplus(a-1) \mathcal{O} \oplus b \mathbb{Z}\left[\mathbb{Z}_{p}\right] \oplus c I d,
$$

where $a, b, c$ are non negative integers with $n=a(p-1)+b p+1, \xi_{p}$ is a primitive $p^{\text {th }}$-root of unity, $\mathcal{O}=\mathbb{Z}\left[\xi_{p}\right]$ is the full ring of algebraic integers in the cyclotomic field $\mathbb{Q}\left(\xi_{p}\right)$ and $\mathfrak{a}$ is an ideal in $\mathcal{O}$. If $\mathfrak{a}$ is principal then one can change $\mathfrak{a}$ by the full ring $\mathcal{O}$. As usual $\mathbb{Z}\left[\mathbb{Z}_{p}\right]$ denotes the group ring over $\mathbb{Z}$, and $I d$ is the trivial $\mathbb{Z}_{p}$-module.

The $\mathbb{Z}_{p}$-actions on the modules are given by multiplication by $\xi_{p}$. In the bases $1, \xi_{p}, \ldots, \xi_{p}^{p-1}$ of $\mathbb{Z}\left[\mathbb{Z}_{p}\right]$ and $1, \xi_{p}, \ldots, \xi_{p}^{p-2}$ of $\mathcal{O}$, the action of the generator is represented, in matrix notation, respectively by

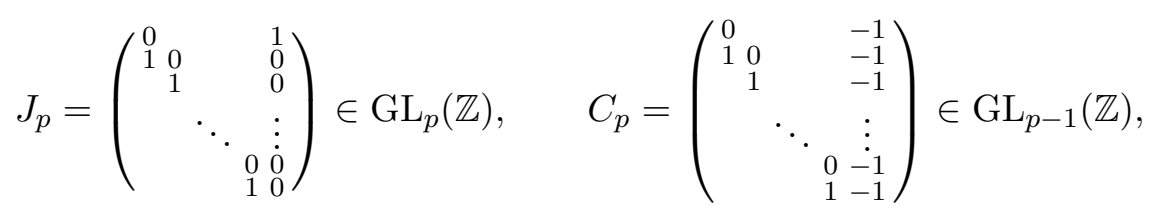

Clearly, we have that $n_{J}=1$ and $n_{C}=0$ (see (1.1)).

It is known (see [6]) that any $\mathbb{Z}_{p}$-manifold is of the form $M=\Gamma \backslash \mathbb{R}^{n}$ where $\Gamma=\left\langle B L_{\frac{e_{n}}{p}}, \Lambda\right\rangle$ is torsion-free, $B \in \mathrm{O}(n)$ of order $p, B e_{n}=e_{n}$ and $\Lambda$ as given in $(2.1)$, is $B$-stable. Furthermore, the torsion-free condition on $\Gamma$ imposes the restriction $c>0$ on the holonomy action.

Recall that we are looking for $n$-dimensional $\mathbb{Z}_{p}$-manifolds having asymmetric Dirac spectrum. By Corollary 2.6 in [17], we have that $\eta(s) \equiv 0$ unless $n_{B}=1$ for $\gamma=B L_{\frac{e_{n}}{p}}$, the generator of $F$. Hence, since $n_{B}=b+c$ and $c>0$, we have that $n_{B}=1$ if and only if $b=0$ and $c=1$. Thus, from now on, we will assume that $\Lambda$ is an orthogonal sum

$$
\tilde{\Lambda}_{p, a}^{\mathfrak{a}}:=\mathfrak{a} \oplus \underbrace{\mathcal{O} \oplus \cdots \oplus \mathcal{O}}_{a-1} \oplus \mathbb{Z} e_{n} .
$$


We set $\Lambda_{1}:=\mathfrak{a} \oplus \underbrace{\mathcal{O} \oplus \cdots \oplus \mathcal{O}}_{a-1}=\left(\mathbb{Z} e_{n}\right)^{\perp}$.

We thus consider the torsion-free group

$$
\tilde{\Gamma}_{p, a}^{\mathfrak{a}}=\left\langle C L_{\frac{e_{n}}{p}}, \tilde{\Lambda}_{p, a}^{\mathfrak{a}}\right\rangle \subset \operatorname{Aff}(n), C=\operatorname{diag}(\underbrace{C_{p}, \ldots, C_{p}}_{a}, 1) \in \mathrm{GL}_{n}(\mathbb{Z}) .
$$

The group $\tilde{\Gamma}_{p, a}^{\mathfrak{a}}$ is not contained in $\mathrm{I}\left(\mathbb{R}^{n}\right)$, however it can be conjugated into a Bieberbach group. Using that the eigenvalues in each block of $\Lambda_{1}$ are exactly the primitive roots of unity, one shows that there exists $A \in \mathrm{GL}_{n}(\mathbb{R})$ such that $B=A C A^{-1} \in \mathrm{SO}(n)$ with $B=\operatorname{diag}(\underbrace{B_{p}, \ldots, B_{p}}_{a}, 1)$ and

$$
B_{p}=\left(\begin{array}{cccc}
\cos \left(\frac{2 \pi}{p}\right) & \sin \left(\frac{2 \pi}{p}\right) & & \\
-\sin \left(\frac{2 \pi}{p}\right) \cos \left(\frac{2 \pi}{p}\right) & & \\
& \ddots & \\
& & \cos \left(\left(\frac{p-1}{2}\right) \frac{2 \pi}{p}\right) & \sin \left(\left(\frac{p-1}{2}\right) \frac{2 \pi}{p}\right) \\
& & -\sin \left(\left(\frac{p-1}{2}\right) \frac{2 \pi}{p}\right) \cos \left(\left(\frac{p-1}{2}\right) \frac{2 \pi}{p}\right)
\end{array}\right) .
$$

Now, if we set $\Gamma_{p, a}^{\mathfrak{a}}:=A \tilde{\Gamma}_{p, a}^{\mathfrak{a}} A^{-1}$, then $\Gamma_{p, a}^{\mathfrak{a}}=\left\langle\gamma, \Lambda_{p, a}^{\mathfrak{a}}\right\rangle \subset \mathrm{I}\left(\mathbb{R}^{n}\right)$, with $\gamma=B L \frac{e_{n}}{p}$ and $\Lambda_{p, a}^{\mathfrak{a}}=A \tilde{\Lambda}_{p, a}^{\mathfrak{a}}$. In this way, we get an orientable Riemannian $n$-manifold

$$
M_{p, a}^{\mathfrak{a}}:=\Gamma_{p, a}^{\mathfrak{a}} \backslash \mathbb{R}^{n}
$$

which is the most general $\mathbb{Z}_{p}$-manifold which may possibly have spectral asymmetry for the Dirac operator $D$. These manifolds correspond to the so called exceptional Bieberbach groups, in the terminology of [7].

Set $p=2 q+1$. Since $n=2 m+1=4 r+3$ and $n=a(p-1)+1$, we have that $m=q a$ is odd, hence $a$ and $q$ are both odd and $p=4 t+3$.

We now deal with the spin structures. It is known that every $F$-manifold with $|F|$ odd is spin (see $[\mathbf{2 2}]$, Corollary 1.3). In [21], the case of $\mathbb{Z}_{p}$-manifolds with $p$ is odd (not necessarily prime) and $n=p$ (i.e. $a=1$ ) is considered, showing the existence of two spin structures. In the next proposition we give the spin structures on the manifolds $M_{p, a}^{\mathfrak{a}}$ defined above. 
Proposition 2.1. Let $p=2 q+1=4 t+3$ be prime. Then $M_{p, a}^{\mathfrak{a}}$ (see (2.4)) has exactly two spin structures $\varepsilon_{h}, h=1,2$, given by

$$
\begin{gathered}
\varepsilon_{h}\left(L_{\lambda}\right)=1 \text { for any } \lambda \in\left(\mathbb{Z} e_{n}\right)^{\perp}, \quad \varepsilon_{h}\left(L_{e_{n}}\right)=(-1)^{h+1}, \\
\varepsilon_{h}(\gamma)=(-1)^{t+h} x_{a}\left(\frac{\pi}{p}, \frac{2 \pi}{p}, \ldots, \frac{q \pi}{p}\right),
\end{gathered}
$$

in the notation of (1.3) and (1.5).

Proof. For simplicity, in the proof we will write $\Lambda, \tilde{\Lambda}, \Gamma, \tilde{\Gamma}$ instead of $\Lambda_{p, a}^{\mathfrak{a}}, \tilde{\Lambda}_{p, a}^{\mathfrak{a}}$, $\Gamma_{p, a}^{\mathfrak{a}}, \tilde{\Gamma}_{p, a}^{\mathfrak{a}}$, respectively.

Assume $\varepsilon$ is a group homomorphism $\Gamma \rightarrow \operatorname{Spin}(n)$ as in (1.8) such that $\mu \circ \varepsilon=r$. Then $\varepsilon$ is determined by the action on $\Lambda$ and on $\gamma=B L_{\frac{e_{n}}{p}}$. We have $\varepsilon\left(L_{\lambda}\right) \in$ $\{ \pm 1\}$, for $\lambda \in \Lambda$ and, in the notation of $(1.5), \varepsilon(\gamma)=\sigma x_{a}\left(\frac{\pi}{p}, \frac{2 \pi}{p}, \ldots, \frac{q \pi}{p}\right)$ with $\sigma \in\{ \pm 1\}$.

Now, since $\gamma^{p} \in L_{\Lambda}$, the character $\varepsilon_{\mid \Lambda}$ satisfies the following conditions

(1) $\quad \varepsilon\left(\gamma^{p}\right)=\left(\sigma x_{a}\left(\frac{\pi}{p}, \frac{2 \pi}{p}, \ldots, \frac{q \pi}{p}\right)\right)^{p}$

$$
\text { (2) } \varepsilon\left(L_{(B-I d) \lambda}\right)=1, \quad \text { for any } \lambda \in \Lambda \text {. }
$$

Conversely, if $\delta \in \operatorname{Hom}(\Lambda,\{ \pm 1\})$ verifies conditions (1) and (2) then $\delta$ extends to a spin structure $\varepsilon$ on $\Gamma([\mathbf{1 9}]$, Proposition 2.2, see also $[\mathbf{1 6}])$.

To determine restrictions on $\varepsilon$ acting on $\left(\mathbb{Z} e_{n}\right)^{\perp}$, we use condition $(2)$ and the integral matrix $C$. For this, define $\tilde{\varepsilon}: \tilde{\Gamma} \rightarrow \operatorname{Spin}(n)$ by $\tilde{\varepsilon}=\varepsilon \circ I_{A}$ where $I_{A}$ is conjugation by $A$. Since $\varepsilon\left(L_{(B-I d) \Lambda}\right)=\varepsilon\left(A L_{(C-I d)} \tilde{\Lambda}^{-1}\right)=\tilde{\varepsilon}\left(L_{(C-I d) \tilde{\Lambda}}\right)$ we have that

$$
\varepsilon\left(L_{(B-I d) \Lambda}\right)=1 \quad \text { if and only if } \quad \tilde{\varepsilon}\left(L_{(C-I d) \tilde{\Lambda}}\right)=1 .
$$

Now for any summand of type $\mathcal{O}$ in (2.2), there is a $\mathbb{Z}$-basis of the form $\left\{e, \xi_{p} e, \ldots, \xi_{p}^{p-2} e\right\}$. Hence by condition (2) we must have

$$
1=\tilde{\varepsilon}\left(\xi_{p} e-e\right)=\cdots=\tilde{\varepsilon}\left(\xi_{p}^{p-2} e-\xi_{p}^{p-3} e\right)=\tilde{\varepsilon}\left(\xi_{p}^{p-1} e-\xi_{p}^{p-2} e\right) .
$$

Thus

$$
\tilde{\varepsilon}(e)=\tilde{\varepsilon}\left(\xi_{p} e\right)=\cdots=\tilde{\varepsilon}\left(\xi_{p}^{p-2} e\right)=\prod_{j=0}^{p-2} \tilde{\varepsilon}\left(\xi_{p}^{j} e\right),
$$

which implies $\tilde{\varepsilon}(e)^{p-2}=1$, and hence $\tilde{\varepsilon}(e)=1$ since $p$ is odd. Therefore, $\tilde{\varepsilon}\left(\xi_{p}^{j} e\right)=$ 1 for every $0 \leq j \leq p-2$ and thus $\tilde{\varepsilon}(\lambda)=1$ for any $\lambda \in \mathcal{O} \oplus \cdots \oplus \mathcal{O}$. 
Now, given a summand of type $\mathfrak{a}$, there exist $e_{1}, e_{2} \in \mathfrak{a}$ such that $\mathfrak{a}=\mathcal{O} e_{1}+\mathcal{O} e_{2}$. By the same argument as in the case of $\mathcal{O}$, we conclude that $\tilde{\varepsilon}_{\mid \mathcal{O} e_{1}}=\tilde{\varepsilon}_{\mid \mathcal{O} e_{2}}=1$. Hence $\tilde{\varepsilon}_{\mid \mathfrak{a}}=1$.

In this way, for any $\lambda \in\left(\mathbb{Z} e_{n}\right)^{\perp}$ we have

$$
\varepsilon\left(L_{\lambda}\right)=\varepsilon\left(L_{A \lambda}\right)=\varepsilon\left(A L_{\lambda} A^{-1}\right)=\tilde{\varepsilon}\left(L_{\lambda}\right)=1 .
$$

This implies the first claim in the proposition.

Relative to the second claim, since $\gamma^{p}=L_{e_{n}}$, condition (1) implies that

$$
\varepsilon\left(L_{e_{n}}\right)=\varepsilon(\gamma)^{p}=\sigma x_{a}\left(\frac{\pi}{p}, \frac{2 \pi}{p}, \ldots, \frac{q \pi}{p}\right)^{p}=\sigma x_{a}(\pi, 2 \pi, \ldots, q \pi)=\sigma(-1)^{t+1}
$$

where we have used (1.4) and the commutativity in $\mathbb{C l}(n)$ of the elements $e_{2 i-1} e_{2 i}$ and $e_{2 j-1} e_{2 j}$ for $i \neq j$.

Hence, $M_{\Gamma}$ has 2 spin structures given by

$$
\varepsilon_{\sigma}=\left(1, \ldots, 1,(-1)^{t+1} \sigma ; \sigma x_{a}\left(\frac{\pi}{p}, \ldots, \frac{q \pi}{p}\right)\right)
$$

with $\sigma \in\{ \pm 1\}$ and the proposition now follows.

\section{ETA SERIES}

We now get into our main task, that is, to explicitly compute the eta series of the manifolds $M_{p, a}^{\mathfrak{a}}$. We begin by giving two identities, possibly known, concerning products of special values of sines which are necessary in the proof of Theorem 3.3. We include the proofs, for completeness.

Lemma 3.1. For any $d \in \mathbb{N}$ one has

$$
\prod_{j=1}^{\left[\frac{d}{2}\right]} \sin \left(\frac{j \pi}{d}\right)=\frac{\sqrt{d}}{2^{\frac{d-1}{2}}}
$$

Proof. We make use of the wellknown identities:

$$
\sin (\pi z)=\frac{\pi}{\Gamma(z) \Gamma(1-z)}, \quad(2 \pi)^{\frac{d-1}{2}} \Gamma(z)=d^{z-\frac{1}{2}} \prod_{j=0}^{d-1} \Gamma\left(\frac{z+j}{d}\right) .
$$

If $d_{0}:=\left[\frac{d}{2}\right]$, it follows that

$$
\prod_{j=1}^{d_{0}} \sin \left(\frac{j \pi}{d}\right)=\frac{\pi^{d_{0}}}{\Gamma\left(\frac{1}{d}\right) \Gamma\left(\frac{d-1}{d}\right) \cdots \Gamma\left(\frac{d_{0}}{d}\right) \Gamma\left(\frac{d-d_{0}}{d}\right)}
$$


and, since $\Gamma(1)=1$, taking $z=1$ in the second expression in (3.2) we get

$$
\prod_{j=1}^{d-1} \Gamma\left(\frac{j}{d}\right)=\frac{(2 \pi)^{\frac{d-1}{2}}}{\sqrt{d}}
$$

Now, if $d=2 d_{0}+1$, expression (3.1) readily follows. If $d=2 d_{0}$, since $\Gamma\left(\frac{d-d_{0}}{d}\right)=$ $\Gamma\left(\frac{d_{0}}{d}\right)$ and $\Gamma\left(\frac{1}{2}\right)=\sqrt{\pi}$, we have the expression

$$
\prod_{j=1}^{d_{0}} \Gamma\left(\frac{j}{d}\right) \Gamma\left(\frac{d-j}{d}\right)=\sqrt{\pi} \prod_{j=1}^{d-1} \Gamma\left(\frac{j}{d}\right)
$$

from which the lemma follows.

Next we consider a similar product as in the previous lemma, but now depending on an integer $k$, for $p$ an odd prime.

Lemma 3.2. Let $k, p \in \mathbb{N}$ and suppose $p$ is an odd prime. Then we have

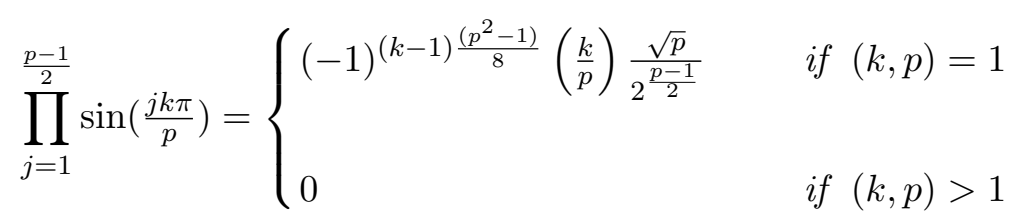

where $(\dot{\bar{p}})$ is the Legendre symbol.

Proof. If $(k, p)=t>1$ then $k=t m, p=t n$ with $m, n \in \mathbb{Z},(m, n)=1$ and $1<n<\frac{p-1}{2}$. Then, for $j=n$ we have that $\frac{j k}{p}=m \in \mathbb{Z}$ and hence $\sin \left(\frac{j k \pi}{p}\right)=0$.

Thus, assume that $(k, p)=1$. For each $1 \leq j \leq p-1$, there exist unique $q_{j}, r_{j} \in \mathbb{Z}$ such that $j k=q_{j} p+r_{j}$ with $0<r_{j}<p$. Since $(k, p)=1$ we have that $r_{1}, r_{2}, \ldots, r_{p-1}$ is a complete system of residues modulo $p$. Furthermore, $\bmod p$,

$$
\{1,2, \ldots, p-1\}=\{k, 2 k, \ldots,(p-1) k\}=\left\{r_{1}, r_{2}, \ldots, r_{p-1}\right\} .
$$

Now, $\operatorname{since} \sin \left(\frac{j k \pi}{p}\right)=(-1)^{q_{j}} \sin \left(\frac{r_{j} \pi}{p}\right)$ and $q_{j}=\left[\frac{j k}{p}\right]$ we get

$$
\prod_{j=1}^{\frac{p-1}{2}} \sin \left(\frac{j k \pi}{p}\right)=\prod_{j=1}^{\frac{p-1}{2}}(-1)^{\left[\frac{j k}{p}\right]} \prod_{j=1}^{\frac{p-1}{2}} \sin \left(\frac{r_{j} \pi}{p}\right)=(-1)^{s_{p}(k)} \prod_{j=1}^{\frac{p-1}{2}} \frac{\pi}{\Gamma\left(\frac{r_{j}}{p}\right) \Gamma\left(\frac{p-r_{j}}{p}\right)},
$$

where $s_{p}(k):=\sum_{j=1}^{\frac{p-1}{2}}\left[\frac{j k}{p}\right]$ and in the last equality we have used (3.2). 
We now compare $r_{j}$ and $r_{p-j}$ for $1 \leq j \leq \frac{p-1}{2}$. We have

$$
(p-j) k=\left(k-q_{j}\right) p-r_{j}=\left(k-q_{j}-1\right) p+\left(p-r_{j}\right) .
$$

Thus $r_{p-j}=p-r_{j}$. This gives $\prod_{j=1}^{\frac{p-1}{2}} \Gamma\left(\frac{r_{j}}{p}\right) \Gamma\left(\frac{p-r_{j}}{p}\right)=\prod_{j=1}^{\frac{p-1}{2}} \Gamma\left(\frac{j}{p}\right) \Gamma\left(\frac{p-j}{p}\right)$ and hence $\prod_{j=1}^{\frac{p-1}{2}} \sin \left(\frac{r_{j} \pi}{p}\right)=\prod_{j=1}^{\frac{p-1}{2}} \sin \left(\frac{j \pi}{p}\right)$. Now, using Lemma 3.1 we arrive at the expression

$$
\prod_{j=1}^{\frac{p-1}{2}} \sin \left(\frac{j k \pi}{p}\right)=(-1)^{s_{p}(k)} \prod_{j=1}^{\frac{p-1}{2}} \sin \left(\frac{j \pi}{p}\right)=(-1)^{s_{p}(k)} \frac{\sqrt{p}}{2^{\frac{p-1}{2}}} .
$$

Finally, by Gauss' lemma (see [1] , Theorems 9.6 and 9.7) we have



which completes the proof.

We are now in a position to compute the eta series and the eta invariants for the Dirac operator $D$. By (0.1), we can write

$$
\eta_{(\Gamma, \varepsilon)}(s)=\frac{1}{(2 \pi)^{s}} \sum_{\mu \in \frac{1}{2 \pi} \mathcal{A}} \frac{d_{\mu}^{+}(\Gamma, \varepsilon)-d_{\mu}^{-}(\Gamma, \varepsilon)}{|\mu|^{s}}
$$

where $d_{\mu}^{ \pm}(\Gamma, \varepsilon)$ are as given in (1.9) and $\mathcal{A}$ denotes the asymmetric spectrum, that is $\mathcal{A}=\left\{\lambda \in \operatorname{Spec}_{D}(M): d_{\lambda} \neq d_{-\lambda}\right\}$.

We recall the $L$-function associated to the quadratic character $\chi=(\dot{\bar{p}})$ modulo $p$ :

$$
L(s, \chi)=\sum_{l=1}^{\infty} \frac{\chi(l)}{l^{s}}=\sum_{l=1}^{\infty} \frac{\left(\frac{l}{p}\right)}{l^{s}} .
$$

We now state one of the main results in this paper.

Theorem 3.3. Let $n=2 m+1=4 r+3, p=2 q+1=4 t+3$ a prime and $a \in \mathbb{N}$ odd such that $n=a(p-1)+1$. Let $\varepsilon_{1}$ and $\varepsilon_{2}$ be the two spin structures of $M_{p, a}^{\mathfrak{a}}$ (see (2.4)) as in Proposition 2.1. Then the corresponding eta series are given by

$$
\begin{gathered}
\eta_{\varepsilon_{1}}(s)=(-1)^{r+t+1} \frac{2 p^{\frac{a-1}{2}}}{(2 \pi)^{s}} L(s, \chi), \\
\eta_{\varepsilon_{2}}(s)=(-1)^{r+t} \frac{2 p^{\frac{a-1}{2}}}{(2 \pi)^{s}}\left(1+\left(\frac{2}{p}\right) 2^{s}\right) L(s, \chi)=\left((-1)^{t+1} 2^{s}-1\right) \eta_{\varepsilon_{1}}(s) .
\end{gathered}
$$


In particular, $\eta_{\varepsilon_{1}}(s)$ and $\eta_{\varepsilon_{2}}(s)$ are everywhere holomorphic in $\mathbb{C}$.

Proof. We need to compute the ingredients in the expression (3.6), so we shall first write down the eigenvalue multiplicity formulas (1.9) in the case at hand. We have that $B, B^{2}, \ldots, B^{p-1} \in F_{1}$. Hence, according to (1.9), if $b_{k}$ is defined by $\gamma^{k}=B^{k} L_{b_{k}}$, we obtain

$$
d_{\mu}^{ \pm}(\Gamma, \varepsilon)=\frac{1}{p}\left(2^{m-1}\left|\Lambda_{\varepsilon, \mu}^{*}\right|+\sum_{k=1}^{p-1} \sum_{u \in\left(\Lambda_{\varepsilon, \mu}^{*}\right)^{B^{k}}} e^{-2 \pi i u \cdot b_{k}} \chi_{L_{n-1}^{ \pm \sigma\left(u, \gamma_{\gamma} k\right.}}\left(x_{\gamma^{k}}\right)\right)
$$

where $2^{m-1}\left|\Lambda_{\varepsilon, \mu}^{*}\right|$ is the contribution of the identity element.

Now, since $\Lambda=\Lambda_{1} \oplus \mathbb{Z} e_{n}$ and $\left(\mathbb{R}^{n}\right)^{B^{k}}=\mathbb{R} e_{n}, 1 \leq k \leq n-1$, we have (see (1.10)) that $\Lambda_{\varepsilon}^{*}=\mathbb{Z} e_{n}$ if $\varepsilon=\varepsilon_{1}$ and $\Lambda_{\varepsilon}^{*}=\left(\mathbb{Z}+\frac{1}{2}\right) e_{n}$ if $\varepsilon=\varepsilon_{2}$. Hence, we get

$$
\left(\Lambda_{\varepsilon, \mu}^{*}\right)^{B^{k}}=\left\{ \pm \mu e_{n}\right\}
$$

with $\mu \in \mathbb{N}$ for $\varepsilon_{1}$ and $\mu \in \mathbb{N}_{0}+\frac{1}{2}$ for $\varepsilon_{2}$.

Furthermore, since $\varepsilon\left(\gamma^{k}\right)=\varepsilon(\gamma)^{k} \in T$, taking

$$
x_{\gamma^{k}}=\varepsilon_{h}(\gamma)^{k}=(-1)^{k(t+h)} x_{a}\left(\frac{\pi k}{p}, \frac{2 \pi k}{p}, \ldots, \frac{q \pi k}{p}\right), \quad(h=1,2)
$$

(see (1.5)) one has that $\sigma\left(e_{n}, x_{\gamma^{k}}\right)=1$ for every $1 \leq k \leq n-1$, by the definition of $\sigma$ (see $[\mathbf{1 7}]$ ).

In this way, using that $b_{k}=\frac{k e_{n}}{p}$, expression (3.10) reduces to

$$
d_{\mu}^{ \pm}\left(\Gamma_{p}, \varepsilon_{h}\right)=\frac{1}{p}\left(2^{m-1}\left|\Lambda_{\varepsilon, \mu}^{*}\right|+\sum_{k=1}^{p-1} S_{k}^{ \pm}(\mu)\right)
$$

where we have put

$$
S_{k}^{ \pm}(\mu):=e^{-\frac{2 \pi i \mu k}{p}} \chi_{L_{n-1}^{ \pm}}\left(x_{\gamma^{k}}\right)+e^{\frac{2 \pi i \mu k}{p}} \chi_{L_{n-1}^{\mp}}\left(x_{\gamma^{k}}\right) .
$$

Now, by (1.7) we have

$$
\chi_{L_{n-1}^{ \pm}}\left(x_{\gamma^{k}}\right)=(-1)^{k(t+h)} 2^{m-1}\left(\left(\prod_{j=1}^{q} \cos \left(\frac{j k \pi}{p}\right)\right)^{a} \pm i^{m}\left(\prod_{j=1}^{q} \sin \left(\frac{j k \pi}{p}\right)\right)^{a}\right) .
$$

Thus, substituting in (3.11) and using that $m=2 r+1$ we see that $S_{k}^{ \pm}(\mu)$ equals

$$
(-1)^{k(t+h)} 2^{m}\left\{\cos \left(\frac{2 k \pi \mu}{p}\right)\left(\prod_{j=1}^{q} \cos \left(\frac{j k \pi}{p}\right)\right)^{a} \pm(-1)^{r} \sin \left(\frac{2 k \pi \mu}{p}\right)\left(\prod_{j=1}^{q} \sin \left(\frac{j k \pi}{p}\right)\right)^{a}\right\} .
$$


hence we obtain

$$
d_{\mu}^{+}\left(\varepsilon_{h}\right)-d_{\mu}^{-}\left(\varepsilon_{h}\right)=\frac{(-1)^{r} 2^{m+1}}{p} \sum_{k=1}^{p-1}(-1)^{k(t+h)} \sin \left(\frac{2 k \pi \mu}{p}\right)\left(\prod_{j=1}^{q} \sin \left(\frac{j k \pi}{p}\right)\right)^{a} .
$$

By Lemma 3.2 , using that $\frac{p^{2}-1}{8} \equiv t+1(\bmod 2)$ and $a q=m$, we arrive at

$$
d_{\mu}^{+}\left(\varepsilon_{h}\right)-d_{\mu}^{-}\left(\varepsilon_{h}\right)=2(-1)^{r+t+1} \frac{(\sqrt{p})^{a}}{p} \sum_{k=1}^{p-1}(-1)^{k(h+1)}\left(\frac{k}{p}\right) \sin \left(\frac{2 k \pi \mu}{p}\right) .
$$

Thus, since

$$
\mu=l \quad \text { for } h=1 \quad \text { and } \quad \mu=l+\frac{1}{2} \quad \text { for } h=2,
$$

with $l \in \mathbb{N}$, using Corollary 5.2 we get

$$
d_{\mu}^{+}\left(\varepsilon_{h}\right)-d_{\mu}^{-}\left(\varepsilon_{h}\right)= \begin{cases}(-1)^{r+t+1} 2 p^{\frac{a-1}{2}}\left(\frac{l}{p}\right) & h=1, \\ (-1)^{r+t} 2 p^{\frac{a-1}{2}}\left(\frac{q-l}{p}\right) & h=2 .\end{cases}
$$

By substituting (3.12) into (3.6), we get

$$
\eta_{\varepsilon_{1}}(s)=(-1)^{r+t+1} \frac{2 p^{\frac{a-1}{2}}}{(2 \pi)^{s}} \sum_{l=1}^{\infty} \frac{\left(\frac{l}{p}\right)}{l^{s}}
$$

so (3.8) holds, and furthermore

$$
\eta_{\varepsilon_{2}}(s)=(-1)^{r+t} \frac{2 p^{\frac{a-1}{2}}}{(2 \pi)^{s}} \sum_{l=0}^{\infty} \frac{\left(\frac{q-l}{p}\right)}{\left(l+\frac{1}{2}\right)^{s}} .
$$

For the second eta series, note that $f_{p}(l)=\left(\frac{q-l}{p}\right)$ is a $p$-periodic function. Thus, we have

$$
\begin{aligned}
\left(\frac{q-l}{p}\right) & =\left(\frac{4}{p}\right)\left(\frac{q-l}{p}\right)=\left(\frac{2}{p}\right)\left(\frac{2 q-2 l}{p}\right)=\left(\frac{2}{p}\right)\left(\frac{p-(2 l+1)}{p}\right) \\
& =\left(\frac{-1}{p}\right)\left(\frac{2}{p}\right)\left(\frac{2 l+1}{p}\right)=-\left(\frac{2}{p}\right)\left(\frac{2 l+1}{p}\right)
\end{aligned}
$$

since $\left(\frac{-1}{p}\right)=-1$ for $p \equiv 3(\bmod 4)$.

Now, splitting sums we have

$$
L(s, \chi)=\sum_{l=1}^{\infty} \frac{\left(\frac{l}{p}\right)}{l^{s}}=\sum_{l \text { even }} \frac{\left(\frac{l}{p}\right)}{l^{s}}+\sum_{l \text { odd }} \frac{\left(\frac{l}{p}\right)}{l^{s}}=: L_{e}(s, \chi)+L_{o}(s, \chi) .
$$


In this way, we get

$$
\sum_{l=0}^{\infty} \frac{\left(\frac{q-l}{p}\right)}{\left(l+\frac{1}{2}\right)^{s}}=-\left(\frac{2}{p}\right) 2^{s} \sum_{l=0}^{\infty} \frac{\left(\frac{2 l+1}{p}\right)}{(2 l+1)^{s}}=-\left(\frac{2}{p}\right) 2^{s} L_{o}(s, \chi) .
$$

Since by definition, $L_{o}(s, \chi)=L(s, \chi)-L_{e}(s, \chi)$, and

$$
L_{e}(s, \chi)=\sum_{l=1}^{\infty} \frac{\left(\frac{2 l}{p}\right)}{(2 l)^{s}}=\frac{\left(\frac{2}{p}\right)}{2^{s}} \sum_{l=1}^{\infty} \frac{\left(\frac{l}{p}\right)}{l^{s}}=\left(\frac{2}{p}\right) 2^{-s} L(s, \chi)
$$

we have that

$$
L_{o}(s, \chi)=\left(1-\left(\frac{2}{p}\right) 2^{-s}\right) L(s, \chi) .
$$

By substituting (3.15) in (3.14) and using (3.16), and the fact that $\left(\frac{2}{p}\right)=$ $(-1)^{\frac{p^{2}-1}{8}}=(-1)^{t+1}$, we finally obtain (3.9), and the theorem follows.

Corollary 3.4. The eta series of $M_{a, p}^{\mathfrak{a}}$ (see (2.4)) can be written in the following way

$$
\eta_{\varepsilon_{1}}(s)=(-1)^{r+t+1} \frac{2 p^{\frac{a-1}{2}}}{(2 \pi p)^{s}} \sum_{j=1}^{q}\left(\frac{j}{p}\right)\left(\zeta\left(s, \frac{j}{p}\right)-\zeta\left(s, 1-\frac{j}{p}\right)\right),
$$

$$
\eta_{\varepsilon_{2}}(s)=(-1)^{r+t} \frac{2 p^{\frac{a-1}{2}}}{(2 \pi p)^{s}} \sum_{j=0}^{q}\left(\frac{q-j}{p}\right)\left(\zeta\left(s, \frac{2 j+1}{2 p}\right)-\zeta\left(s, 1-\frac{2 j+1}{2 p}\right)\right),
$$

where, if $\alpha \in(0,1], \zeta(s, \alpha)=\sum_{l=0}^{\infty} \frac{1}{(l+\alpha)^{s}}$, the Hurwitz zeta function. The functions $\eta_{\varepsilon_{1}}(s)$ and $\eta_{\varepsilon_{2}}(s)$ are entire.

Proof. Starting from expressions (3.13) and (3.14) and setting $l=p t+j$, with $0 \leq j \leq p-1$, we get

$$
\begin{aligned}
\eta_{\varepsilon_{1}}(s) & =(-1)^{r+t+1} \frac{2 p^{\frac{a-1}{2}}}{(2 \pi p)^{s}} \sum_{j=1}^{p-1}\left(\frac{j}{p}\right) \sum_{t=1}^{\infty} \frac{1}{\left(t+\frac{j}{p}\right)^{s}} \\
& =(-1)^{r+t+1} \frac{2 p^{\frac{a-1}{2}}}{(2 \pi p)^{s}} \sum_{j=1}^{p-1}\left(\frac{j}{p}\right) \zeta\left(s, \frac{j}{p}\right) .
\end{aligned}
$$

Proceeding similarly, we get

$$
\eta_{\varepsilon_{2}}(s)=(-1)^{r+t} \frac{2 p^{\frac{a-1}{2}}}{(2 \pi p)^{s}} \sum_{j=0}^{p-1}\left(\frac{q-j}{p}\right) \zeta\left(s, \frac{2 j+1}{2 p}\right) .
$$


Now, since $\left(\frac{-1}{p}\right)=-1$ for $p \equiv 3(\bmod 4)$, we have

$$
\left(\frac{p-l}{p}\right)=\left(\frac{-l}{p}\right)=\left(\frac{-1}{p}\right)\left(\frac{l}{p}\right)=-\left(\frac{l}{p}\right) .
$$

Also, if $l^{\prime}=p-(l+1)$ with $0 \leq l \leq q$ then $q-l^{\prime}=-(q-l)$. Thus

$$
\left(\frac{q-l^{\prime}}{p}\right)=\left(\frac{-1}{p}\right)\left(\frac{q-l}{p}\right)=-\left(\frac{q-l}{p}\right) .
$$

From these relations, together with (3.18) and (3.19), we obtain expressions (3.17) in the statement. The last assertion follows from the fact that, for each $\alpha, \zeta(s, \alpha)$ is a meromorphic function on $\mathbb{C}$ with a simple pole at $s=1$, with residue 1 .

\section{Eta inVARIANTS AND ClASS NUMBERS}

Here we compute the $\eta$-invariants of any $\mathbb{Z}_{p}$-manifold, $p$ an odd prime. We shall see, as a consequence of Theorem 3.3, that they admit simple expressions in terms of the class number $h_{-p}$ of the quadratic field $\mathbb{Q}(\sqrt{-p})$ (see Introduction).

Theorem 4.1. Let $n=a(p-1)+1$ with a odd and $p$ and odd prime. The $\eta$ invariants of $M_{p, a}^{\mathfrak{a}}$ (see (2.4)) are given as follows. If $p=3$,

$$
\eta_{\varepsilon_{1}}=(-1)^{r+1} 2 \cdot 3^{\frac{a-3}{2}} \quad \text { and } \quad \eta_{\varepsilon_{2}}=(-1)^{r} 4 \cdot 3^{\frac{a-3}{2}} .
$$

If $p \geq 7$,

$$
\begin{gathered}
\eta_{\varepsilon_{1}}=(-1)^{r+t} 2 p^{\frac{a-3}{2}} \sum_{k=1}^{p-1} k\left(\frac{k}{p}\right)=(-1)^{r+t+1} 2 p^{\frac{a-1}{2}} h_{-p} \\
\eta_{\varepsilon_{2}}=\left((-1)^{t+1}-1\right) \eta_{\varepsilon_{1}}= \begin{cases}0 & p \equiv 7(\bmod 8) \\
(-1)^{r+t} 4 p^{\frac{a-1}{2}} h_{-p} & p \equiv 3(\bmod 8)\end{cases}
\end{gathered}
$$

where $h_{-p}$ denotes the class number of the quadratic field $\mathbb{Q}(\sqrt{-p})$.

Proof. We shall use Theorem 3.3. By (3.8), since for a non-trivial character $\chi$, $L(0, \chi)=-\frac{1}{p} \sum_{l=0}^{p-1} l \chi(l)$ (see $[\mathbf{1}]$, pp. 268), we get the first equality in (4.1). Now, by (3.9), the first equality in (4.2) is obvious. (This also follows by evaluating the expressions (3.18) and (3.19) at $s=0$ and using that $\zeta(0, \alpha)=\frac{1}{2}-\alpha$ and $\left.\sum_{l=1}^{p-1}\left(\frac{l}{p}\right)=0\right)$. 
To verify the remaining identities we will use the Dirichlet's class number formula for an odd prime $p \equiv 3(\bmod 4)$ (see for instance [4], pp. 377) given by

$$
\frac{h_{-p}}{\omega_{-p}}=-\frac{1}{2 p} \sum_{l=0}^{p-1} l\left(\frac{l}{p}\right)
$$

where $\omega_{-p}$ is the number of roots of unity in $\mathbb{Q}(\sqrt{-p})$. Note that $\omega_{-3}=6$ and $\omega_{-p}=2$ for $p \geq 7$.

Thus, by (4.3) and the first equality in (4.1) we have

$$
\eta_{\varepsilon_{1}}=(-1)^{r+t} 2 p^{\frac{a-1}{2}}\left(\frac{1}{p} \sum_{l=0}^{p-1} l\left(\frac{l}{p}\right)\right)=(-1)^{r+t+1} 4 p^{\frac{a-1}{2}} \frac{h_{-p}}{\omega_{-p}}
$$

from which (4.1) follows directly for $p \geq 7$ and, for $p=3$, we see that $\eta_{\varepsilon_{1}}=$ $(-1)^{r+t} 2 \cdot 3^{\frac{a-3}{2}}$ since $h_{-3}=1$.

Finally, introducing the second expression in (4.1) into (4.2) we get $\eta_{\varepsilon_{2}}=$ $(-1)^{r} 4 \cdot 3^{\frac{a-3}{2}}$ for $p=3$, and

$$
\eta_{\varepsilon_{2}}= \begin{cases}0 & p \equiv 7(\bmod 8) \\ -2 \eta_{\varepsilon_{1}} & p \equiv 3(\bmod 8)\end{cases}
$$

for $p \geq 7$, and the theorem follows.

Remark 4.2. (i) In [18], Pfäffle computes the eta invariants of all 3-dimensional compact flat manifolds. In [21], the authors consider the case of a family of $\mathbb{Z}_{n^{-}}$ manifolds of dimension $n$, arriving at an expression of the eta invariant in terms of solutions of certain congruences. Also, in [16], Proposition 3.4, the eta series and eta invariants are computed for all $\mathbb{Z}_{2}^{k}$-manifolds.

(ii) Note that, from the expressions in the theorem, we see that $\eta \in 2 \mathbb{Z}$ except in the case when $p=3$ and $a=1$. In this case, for the 3 -dimensional $\mathbb{Z}_{3}$-manifold we have $\eta_{\varepsilon_{1}}=-\frac{2}{3}$ and $\eta_{\varepsilon_{2}}=\frac{4}{3}$. These values coincide with those obtained in [18] and [17]. We note that always $\eta_{\varepsilon_{1}}-\eta_{\varepsilon_{2}} \in 2 \mathbb{Z}$ and, if $p \equiv 3(\bmod 8)$, then $\eta_{\varepsilon_{2}}=-2 \eta_{\varepsilon_{1}}$.

In [17], Theorem 5.1, we have derived an expression for the eta invariants $\eta_{\varepsilon_{1}}$ and $\eta_{\varepsilon_{2}}$ in the case $n=p$, involving cotangent or cosecant sums, together with Legendre symbols. Here, for any $n=a(p-1)+1$, we will obtain, in a different way, a generalization of such expressions. 
Proposition 4.3. The $\eta$-invariants of $M_{p, a}^{\mathfrak{a}}$ can be written:

$$
\begin{aligned}
& \eta_{\varepsilon_{1}}=(-1)^{r+t+1} p^{\frac{a-2}{2}} \sum_{k=1}^{p-1}\left(\frac{k}{p}\right) \cot \left(\frac{\pi k}{p}\right)=(-1)^{r+t+1} p^{\frac{a-2}{2}} \sum_{k=1}^{p-1} \cot \left(\frac{\pi k^{2}}{p}\right), \\
& \eta_{\varepsilon_{2}}=(-1)^{r+t+1} p^{\frac{a-2}{2}} \sum_{k=1}^{p-1}(-1)^{k}\left(\frac{k}{p}\right) \csc \left(\frac{\pi k}{p}\right) .
\end{aligned}
$$

Note. We point out that by $(4.2), \eta_{\varepsilon_{2}}$ can also be written in terms of cotangents. On the other hand, $\eta_{\varepsilon_{1}}$ has an expression in terms of cosecants only for $p \equiv 3(8)$.

Proof. From (3.18), by using that $\zeta(0, \alpha)=\frac{1}{2}-\alpha$ (see [1] , pp. 268), we have that $\eta_{\varepsilon_{1}}=(-1)^{r+t+1} 2 p^{\frac{a-1}{2}} \sum_{j=1}^{p-1}\left(\frac{j}{p}\right)\left(\frac{1}{2}-\frac{j}{p}\right)$. Now, consider the sawtooth function defined by

$$
((x))= \begin{cases}x-\lfloor x\rfloor-\frac{1}{2} & \text { if } x \in \mathbb{R} \backslash \mathbb{Z} \\ 0 & \text { if } x \in \mathbb{Z} .\end{cases}
$$

Note that since $0<\frac{j}{p}<1$ and $\frac{j}{p} \notin \mathbb{Z}$ we have $\left(\left(\frac{j}{p}\right)\right)=\frac{j}{p}-\frac{1}{2}$ and thus

$$
\eta_{\varepsilon_{1}}=(-1)^{r+t} 2 p^{\frac{a-1}{2}} \sum_{j=1}^{p-1}\left(\frac{j}{p}\right)\left(\left(\frac{j}{p}\right)\right) .
$$

Also, $((x))$ is odd and 1-periodic. Thus, as a function of $k,\left(\left(\frac{k}{p}\right)\right)$ has a finite Fourier expansion given by (see [12], pp. 98-99)

$$
\left(\left(\frac{j}{p}\right)\right)=-\frac{1}{2 p} \sum_{k=1}^{p-1} \sin \left(\frac{2 \pi k j}{p}\right) \cot \left(\frac{\pi k}{p}\right) .
$$

Substituting (4.7) into (4.6) we get

$$
\eta_{\varepsilon_{1}}=(-1)^{r+t+1} p^{\frac{a-3}{2}} \sum_{k=1}^{p-1}\left(\sum_{j=1}^{p-1}\left(\frac{j}{p}\right) \sin \left(\frac{2 \pi k j}{p}\right)\right) \cot \left(\frac{\pi k}{p}\right)
$$

and, by Corollary 5.2, the first identity in (4.4) follows.

Now, using the identity $\sum_{k=1}^{p-1} \cot \left(\frac{\pi k^{2}}{p}\right)=-\frac{2}{\sqrt{p}} \sum_{k=1}^{p-1} k\left(\frac{k}{p}\right)$ (see [4], pp. 42) and (4.1) we get the second identity in (4.4).

On the other hand, from (3.19) we have that

(4.8) $\eta_{\varepsilon_{2}}(0)=(-1)^{r+t} 2 p^{\frac{a-1}{2}} \sum_{j=0}^{p-1}\left(\frac{q-j}{p}\right)\left(\frac{1}{2}-\frac{2 j+1}{2 p}\right)=(-1)^{r+t+1} 2 p^{\frac{a-3}{2}} \sum_{j=0}^{p-1} j\left(\frac{q-j}{p}\right)$ 
where we have used that $\sum_{j=0}^{p-1}\left(\frac{q-j}{p}\right)=\sum_{j=0}^{p-1}\left(\frac{j}{p}\right)=0$. Using Corollary 5.2 again, we have

$$
\left(\frac{q-j}{p}\right)=-\frac{1}{\sqrt{p}} \sum_{k=1}^{p-1}(-1)^{k}\left(\frac{k}{p}\right) \sin \left(\frac{(2 j+1) \pi k}{p}\right) .
$$

Thus, substituting this expression in (4.8) we obtain

$$
\eta_{\varepsilon_{2}}=(-1)^{r+t} 2 p^{\frac{a-4}{2}} \sum_{k=1}^{p-1}(-1)^{k}\left(\frac{k}{p}\right) \sum_{j=0}^{p-1} j \sin \left(\frac{(2 j+1) \pi k}{p}\right) .
$$

Now, using the identity

$$
\sum_{j=0}^{p-1} j \sin \left(\frac{(2 j+1) \pi k}{p}\right)=-\frac{p}{2} \csc \left(\frac{\pi k}{p}\right)
$$

(see [17], pp. 33) we finally obtain (4.5), and thus the proposition follows.

An alternative approach. In this subsection, we will show how to get expressions for $\eta_{\varepsilon_{1}}, \eta_{\varepsilon_{2}}$ in Theorem 4.1, involving class numbers, directly from the trigonometric expressions in Proposition 4.3. In particular, this gives an alternative way to obtain (4.1) and (4.2).

Let $D$ be a negative discriminant. If $\chi_{D}$ is the Kronecker symbol, the Dirichlet $L$-function

$$
L\left(s, \chi_{D}\right):=\sum_{n=1}^{\infty} \frac{\chi_{D}(n)}{n^{s}}, \quad \operatorname{Re}(s)>1,
$$

can be analytically continued to the whole complex plane to an entire function. We will use the class number formula (see [24], Satz 5, pp. 72)

$$
2 \pi h_{D}=w_{D} \sqrt{|D|} L\left(1, \chi_{D}\right)
$$

where $w_{D}$ denote the number of roots of unity lying in the quadratic imaginary field $\mathbb{Q}(\sqrt{D})$. We note that $w_{D}=6,4$ for $D=-3,-4$ respectively and $w_{D}=2$ for $D<-4$.

We will make use of the following classical class number formulas. We include a proof for completeness (see also [5]). 
Theorem 4.4. (cotangent and cosecant formulas) Suppose that $D$ is odd and $\chi_{D}(-1)=-1$. Then we have

$$
\begin{gathered}
\frac{1}{\sqrt{|D|}} \sum_{k \bmod D} \chi_{D}(k) \cot \left(\frac{\pi k}{|D|}\right)=\frac{4 h_{D}}{w_{D}} \\
\frac{1}{\sqrt{|D|}} \sum_{k \bmod D}(-1)^{k} \chi_{D}(k) \csc \left(\frac{\pi k}{|D|}\right)=\left\{\begin{array}{cc}
-\frac{8 h_{D}}{w_{D}} & \text { if } \chi_{D}(2)=-1 \\
0 & \text { if } \chi_{D}(2)=1 .
\end{array}\right.
\end{gathered}
$$

If $\chi_{D}(-1)=1$ the cotangent expression in (4.11) is obviously 0 .

Proof. We will use the well-known expansion $\pi \cot (\pi z)=\sum_{n \in \mathbb{Z}}^{\prime} \frac{1}{z-n}$, where the summation collects the summands for $n,-n$, with $n \neq 0$. This convention is used throughout and makes the following calculation rigorous. We have

$$
\begin{aligned}
\pi \sum_{k \bmod D} \chi_{D}(k) \cot \left(\frac{\pi k}{|D|}\right) & =\sum_{m \in \mathbb{Z}} \sum_{k \bmod D} \frac{|D|}{k-m|D|} \chi_{D}(k) \\
& =|D| \sum_{n \in \mathbb{Z}}^{\prime} \frac{\chi_{D}(n)}{n}=\frac{4 \pi \sqrt{|D|} h_{D}}{w_{D}},
\end{aligned}
$$

where we have used (4.9), (4.10) and $\chi_{D}(-1)=-1$, from which (4.11) follows.

By the expansion $\pi \csc (\pi z)=\sum_{n \in \mathbb{Z}}^{\prime} \frac{(-1)^{n}}{z-n}$, the expression

$$
\pi \sum_{k \bmod D}(-1)^{k} \chi_{D}(k) \csc \left(\frac{\pi k}{|D|}\right)
$$

equals

$$
\begin{gathered}
\sum_{m \in \mathbb{Z}}^{\prime} \sum_{k \bmod D} \frac{|D|}{k-2 m|D|} \chi_{D}(k)-\sum_{m \in \mathbb{Z}}^{\prime} \sum_{k \bmod D} \frac{|D|}{k-(2 m+1)|D|} \chi_{D}(k)= \\
2 \sum_{m \in \mathbb{Z}} \sum_{k \bmod D} \frac{|D|}{k-2 m|D|} \chi_{D}(k)-\sum_{n \in \mathbb{Z}}^{\prime} \sum_{k \bmod D} \frac{|D|}{k-n|D|} \chi_{D}(k) .
\end{gathered}
$$

The expression $(-1)^{k} \chi_{D}(k) \csc \left(\frac{\pi k}{|D|}\right)$ depends only on the residue class of $k$ mod $D$. Since even numbers represent all residue classes we can replace $k$ by $2 k$ in the above expression, hence by (4.9) we have

$$
\sum_{m \in \mathbb{Z}}^{\prime} \sum_{k \bmod D} \frac{|D|}{2 k-2 m|D|} \chi_{D}(2 k)=|D| \chi_{D}(2) L\left(1, \chi_{D}\right) .
$$


Thus, the previous calculations together with (4.10) imply

$$
\pi \sum_{k \bmod D}(-1)^{k} \chi_{D}(k) \csc \left(\frac{\pi k}{|D|}\right)=4 \pi\left(\chi_{D}(2)-1\right) \sqrt{|D|} \frac{h_{D}}{w_{D}}
$$

and thus (4.12) follows.

We thus see that, starting from expressions (4.4) and (4.5), and using (4.11) and (4.12) with $D=-p$, we arrive at an alternative proof of formulas (4.1) and (4.2) for the $\eta$-invariants in terms of class numbers.

Remark 4.5. The main approach in this paper applies to flat manifolds with more general holonomy groups. On the other hand, since there is no known classification of such manifolds even in the case of cyclic holonomy groups (except when the order is $p$ or $p^{2}, p$ prime) a general result for the eta invariant seems out of reach. We plan to deal with the computation of eta invariants in a more general setting in a continuation of this paper.

\section{Appendix: Modified Gauss sums.}

In this section we prove some identities involving sums of special values of sines, which are slight modifications of Gauss sums and which have been used in the development of the formulas for the eta series and eta invariants in Sections 3 and 4 .

Consider the character Gauss sum $G(l, p)$ associated to the quadratic Dirichlet character given by the Legendre symbol modulo $p$, and the quadratic Gauss sum $g(l, p)$ modulo $p$, where $l, p \in \mathbb{N}$. As it is known, in the case when $p$ is odd and squarefree, and furthermore $l$ and $p$ are coprime, these two sums coincide. Namely,

$$
G(l, p):=\sum_{k=0}^{p-1}\left(\frac{k}{p}\right) e^{\frac{2 \pi i l k}{p}}=\sum_{k=0}^{p-1} e^{\frac{2 \pi i l k^{2}}{p}}=g(l, p) .
$$

However, if $(l, p)>1$ these two sums differ since $G(l, p)=0$ while $g(l, p)=p$. We will need the identities:

$$
g(l, p)=\left(\frac{l}{p}\right) g(1, p)= \begin{cases}\sqrt{p}\left(\frac{l}{p}\right) & p \equiv 1(\bmod 4) \\ i \sqrt{p}\left(\frac{l}{p}\right) & p \equiv 3(\bmod 4) .\end{cases}
$$


We now introduce some variants of $G(l, p)$. Let $l, p \in \mathbb{N}$ with $p$ odd. The alternating Gauss sum, shifted Gauss sum and alternating shifted Gauss sum are respectively defined by

$$
\begin{aligned}
\tilde{G}(l, p) & :=\sum_{k=0}^{p-1}(-1)^{k}\left(\frac{k}{p}\right) e^{\frac{2 \pi i l k}{p}} \\
H(l, p) & :=\sum_{k=0}^{p-1}\left(\frac{k}{p}\right) e^{\frac{(2 l+1) \pi i k}{p}} \\
\tilde{H}(l, p) & :=\sum_{k=0}^{p-1}(-1)^{k}\left(\frac{k}{p}\right) e^{\frac{(2 l+1) \pi i k}{p}} .
\end{aligned}
$$

In the next result we give alternative expressions for these sums.

Theorem 5.1. Let $l \in \mathbb{N}$ and $p=2 q+1$ be an odd prime. If $p \nmid l$, then

$$
\begin{aligned}
& \tilde{G}(l, p)=\frac{-i}{G(1, p)} \sum_{k=1}^{p-1}\left(\frac{k}{p}\right) \tan \left(\frac{(k+l) \pi}{p}\right) \\
& H(l, p)=\frac{i}{G(1, p)} \sum_{k=1}^{p-1}\left(\frac{k}{p}\right) \cot \left(\frac{(2(k+l)+1) \pi}{2 p}\right) \\
& \tilde{H}(l, p)=\frac{1}{G(1, p)}\left(\frac{q-l}{p}\right)
\end{aligned}
$$

On the other hand, if $p \mid l$, all the sums above vanish.

Proof. We use the same method for all three identities, which is simply to multiply by $G(1, p)$ the modified Gauss sum to be computed.

First, consider the product $G(1, p) \tilde{G}(l, p)$. Since for each $k$ fixed, with $1 \leq k \leq$ $p-1$, there is a unique $t=t_{p}(j, k)$ such that $j \equiv t k(\bmod p)$, we have

$$
G(1, p) \tilde{G}(l, p)=\sum_{j, k=0}^{p-1}(-1)^{k}\left(\frac{j}{p}\right)\left(\frac{k}{p}\right) e^{\frac{2 \pi i j}{p}} e^{\frac{2 \pi i l k}{p}}=\sum_{t=0}^{p-1}\left(\frac{t}{p}\right) \sum_{k=0}^{p-1}(-1)^{k} z_{t, l}^{k}
$$

where $z_{t, l}:=e^{\frac{2 \pi i(t+l)}{p}}$. Since $z_{t, l}^{p}=1$ and $z_{t, l} \neq-1$ for any $t, l$, by geometric summation we get $\sum_{k=0}^{p-1}(-1)^{k} z_{t, l}^{k}=\frac{1-z_{t, l}}{1+z_{t, l}}+1$. Thus, by using that $\sum_{t=0}^{p-1}\left(\frac{t}{p}\right)=$ 0 and $\frac{1-e^{i s}}{1+e^{i s}}=-i \tan \left(\frac{s}{2}\right)$, we finally obtain

$$
G(1, p) \tilde{G}(l, p)=\sum_{t=0}^{p-1}\left(\frac{t}{p}\right) \frac{1-z_{t, l}}{1+z_{t, l}}=-i \sum_{t=0}^{p-1}\left(\frac{t}{p}\right) \tan \left(\frac{\pi(t+l)}{p}\right)
$$


from which the first identity in the theorem follows.

Now, consider the product $G(1, p) H(l, p)$. Proceeding as before, we have

$$
G(1, p) H(l, p)=\sum_{t=0}^{p-1}\left(\frac{t}{p}\right) \sum_{t=0}^{p-1} x_{t, l}^{k}=\sum_{t=0}^{p-1}\left(\frac{t}{p}\right) \frac{1+x_{t, l}}{1-x_{t, l}}
$$

where $x_{t, l}=e^{\frac{(2(t+l)+1) \pi i}{p}}$ and where we have used that $x_{t, l}^{p}=-1$ and $x_{t, l} \neq 1$, for any $t, l$. In this way, we get

$$
G(1, p) H(l, p)=i \sum_{t=0}^{p-1}\left(\frac{t}{p}\right) \cot \left(\frac{(2(t+l)+1) \pi}{2 p}\right)
$$

from which the second equality follows.

Finally, we consider the product $G(1, p) \tilde{H}(l, p)$. As before we have

$$
G(1, p) \tilde{H}(l, p)=\sum_{t=1}^{p-1}\left(\frac{t}{p}\right) \sum_{k=1}^{p-1}(-1)^{k} x_{t, l}^{k} .
$$

Note that $x_{t, l}^{k}=-1$ if and only if $p \mid 2(t+l)+1$, that is, if and only if $2(t+l)+1=$ $\alpha p$, with $\alpha$ odd. In this case, $\alpha=1$ or $\alpha=3$. Thus, $t+l=q$ or $t+l=3 q+1$ and hence $t=q-l$ for $l<q$ and $t=3 q-l+1=p+(q-l)$ for $q<l<p-1=2 q$.

Suppose that $l<q$. Since $x_{t, l}^{p}=-1$ and $x_{t, l} \neq 1$, we have that $\sum_{k=0}^{p-1}(-1)^{k} x_{t, l}^{k}=$ 0 . In this way, we get

$$
G(1, p) \tilde{H}(l, p)=\left(\frac{q-l}{p}\right) \sum_{k=0}^{p-1}(-1)^{k}(-1)^{k}=p\left(\frac{q-l}{p}\right)
$$

from which the last equality follows.

In the remaining case, $q<l<p-1$, the computations give the same result since $\left(\frac{p+(q-l)}{p}\right)=\left(\frac{q-l}{p}\right)$, thus Theorem 5.1 follows. 
Corollary 5.2. Let $l \in \mathbb{N}$ and $p$ an odd prime. If $p \nmid l$, then

$$
\begin{aligned}
& \sum_{k=1}^{p-1}\left(\frac{k}{p}\right) \sin \left(\frac{2 l \pi k}{p}\right)=\left\{\begin{array}{l}
0 \\
\sqrt{p}\left(\frac{l}{p}\right)
\end{array}\right. \\
& \sum_{k=1}^{p-1}(-1)^{k}\left(\frac{k}{p}\right) \sin \left(\frac{2 l \pi k}{p}\right)=\left\{\begin{array}{cl}
\frac{-1}{\sqrt{p}} \sum_{k=1}^{p-1}\left(\frac{k}{p}\right) \tan \left(\frac{(k+l) \pi}{p}\right) & p \equiv 1(\bmod 4) \\
0 & p \equiv 3(\bmod 4)
\end{array}\right. \\
& \sum_{k=1}^{p-1}\left(\frac{k}{p}\right) \sin \left(\frac{(2 l+1) \pi k}{p}\right)=\left\{\begin{array}{cl}
\frac{1}{\sqrt{p}} \sum_{k=1}^{p-1}\left(\frac{k}{p}\right) \cot \left(\frac{(2(k+l)+1) \pi}{2 p}\right) & p \equiv 1(\bmod 4) \\
0 & p \equiv 3(\bmod 4)
\end{array}\right. \\
& \sum_{k=1}^{p-1}(-1)^{k}\left(\frac{k}{p}\right) \sin \left(\frac{(2 l+1) \pi k}{p}\right)= \begin{cases}0 & p \equiv 1(\bmod 4) \\
-\sqrt{p}\left(\frac{q-l}{p}\right) & p \equiv 3(\bmod 4)\end{cases} \\
& p \equiv 1(\bmod 4) \\
& p \equiv 3(\bmod 4)
\end{aligned}
$$

On the other hand, if $p \mid l$, all these sums vanish.

Proof. Simply note that the left hand side of the expressions in the statement are the imaginary part of different types of Gauss sums, namely they are equal, respectively, to $\operatorname{Im} G(l, p), \operatorname{Im} \tilde{G}(l, p), \operatorname{Im} \tilde{H}(l, p)$ and $\operatorname{Im} \tilde{H}(l, p)$. The result readily follows by using (5.1) and (5.2).

\section{REFERENCES}

[1] T. Apostol, Introduction to analytic number theory, Springer Verlag UTM, New York, 1998.

[2] M. F. Atiyah, V. K. Patodi, I. M. Singer, Spectral asymmetry and Riemannian geometry I, II, III, Math. Proc. Cambridge Philos. Soc. 77, (43-69) 1975; 78 (405-432) 1975; 79, (71-99) 1976.

[3] L. Auslander, H. Szczarba, Characteristic classes of compact solvmanifolds, Annals of Math. 76, 2, (1-8) 1962.

[4] B. Berndt, R. Evans, K. Williams, Gauss and Jacobi sums, Canadian Math. Soc. Series, Vol. 21, Wiley-Interscience, 1998.

[5] B. Berndt, A. Zaharescu, Finite trigonometric sums and class numbers, Math. Annalen Vol. 330, 3, (551-575) 2004.

[6] L. Charlap, Compact flat Riemannian manifolds I, Ann. of Math. 81, (15-30) 1965.

[7] L. Charlap, Bieberbach groups and flat manifolds, Springer Verlag, Universitext, 1988.

[8] T. Friedrich, Die Abhängigkeit des Dirac-Operators von der Spin-Struktur, Coll. Math. XLVII (57-62) 1984.

[9] T. Friedrich, Dirac operator in Riemannian geometry, Amer. Math. Soc. GSM 25, 1997. 
[10] P. B. Gilkey, The Residue of the Global $\eta$ Function at the Origin, Adv. in Math. 40, (290-307) 1981.

[11] P. B. Gilkey, The geometry of spherical space form groups, World Scientific, Series in Pure Mathematics Vol 7, 1989.

[12] F. Hirzebruch, D. Zagier, The Atiyah-Singer Theorem and Elementary Number Theory, Publish or Perish, Math. Lecture Series 3, 1974.

[13] S. M. Im, H. K. Kim, Compact flat manifolds with non-vanishing Stiefel-Whitney classes, Topology and its Applications 96, (267-276) 1999.

[14] H. B. Lawson, M. L. Michelsohn, Spin geometry, Princeton University Press, New Jersey, 1989.

[15] R. Lee, R. H. Szczarba, On the integral Pontrjagin classes of a Riemannian flat manifold, Geom. Dedicata 3, (1-9) 1974.

[16] R. J. Miatello, R. A. Podestá, Spin structures and spectra of $\mathbb{Z}_{2}^{k}$-manifolds, Math. Zeitschrift 247, (319-335) 2004.

[17] R. J. Miatello, R. A. Podestá, The spectrum of twisted Dirac operators on compact flat manifolds, Trans. Amer. Math. Society 358, 10, (4569-4603) 2006.

[18] F. Pfäffle, The Dirac spectrum of Bieberbach manifolds, J. Geom. Phys. 35 (367-385) 2000.

[19] R. A. Podestá, Eta series and eta invariants of $\mathbb{Z}_{4}$-manifolds, Rev. de la Un. Mat. Arg., 46, 1, (31-45) 2005.

[20] I. Reiner, Integral representations of cyclic groups of prime order, Proc. Amer. Math. Soc. 8, (142-145) 1957.

[21] M. Sadowski, A. Szczepanski, Flat manifolds, harmonic spinors and eta invariants, Adv. in Geometry 6, (287-300) 2006.

[22] A. Vasquez, Flat Riemannian manifolds, J. Diff. Geometry 4 (367-382), 1970.

[23] J. Wolf, Spaces of constant curvature, Mc Graw-Hill, New York, 1967.

[24] D. Zagier, Zetafunktionen und quadratischer Zahlkörper, Springer Verlag 1981.

Roberto J. Miatello and Ricardo A. Podestá

FaMAF-CIEM, Universidad Nacional de Córdoba

Ciudad Universitaria, (5000) Córdoba, República Argentina.

E-mail:miatello@mate.uncor.edu

E-mail:podesta@mate.uncor.edu 